\title{
Points of Departure. Understanding Gender Differences in Faculty Turnover Intentions at University of $X$
}

\section{Dr. Robin O. Andreasen, University of Delaware}

Robin O. Andreasen (Ph.D. University of Wisconsin-Madison) is Associate Professor of Linguistics and Cognitive Science. She earned her PhD in philosophy and specializes in philosophy of science, philosophy of social science, and in science and policy. A race and gender scholar, Dr. Andreasen is research director and co-PI for UD's ADVANCE-IT grant.

\section{Dr. Shawna Vican, University of Delaware}

Shawna Vican is an Assistant Professor of Sociology and Criminal Justice at the University of Delaware. She received her Ph.D. in Sociology from Harvard University. An organizational sociologist, Dr. Vican investigates the adoption and implementation of new employment practices and corporate social behaviors. Across her research, Dr. Vican explores how organizational policies and practices, managerial behavior, and workplace culture shape individual career outcomes as well as broader patterns of labor market inequality. Her current research includes a qualitative study of corporate diversity management strategies and a series of mixed-methods projects on diversity in the academic workforce.

\section{Prof. Yvette A Jackson, University of Massachusetts Dartmouth}

Yvette Jackson, Professor of Synthetic Organic Chemistry, recently retired from the University of the West Indies where she was Pro-Vice-Chancellor, Graduate Studies, with responsibility for graduate education across all campuses of the university. Professor Jackson joined the UD ADVANCE institute at UD in September 2017. 


\section{Points of Departure: Understanding Gender Differences in Faculty Turnover at University of $X$}

\section{Introduction}

Career disparities by gender in the STEM fields are well documented.1 Women are underrepresented at most levels in mathematics, the physical sciences, and most fields of engineering [13], [23]. Even in fields where women are well-represented, such as biology or the health sciences, there is a pattern of increased gender disparity with increased professional status. In academia, for example, women's representation is higher in the non-tenured (42\%) than in the tenure-track (37\%) or tenured (28\%) STEM faculty positions [13].2 Data also indicate a decrease in women's representation with an increase in rank. In 2017, for example, women occupied $40 \%$ of the assistant professor, $36 \%$ of the associate professor, and only $24 \%$ of the full professor STEM faculty positions [13].3 Women are also under-represented as department chairs and in other leadership positions [7], [19]. This is so in spite of the fact that the proportion of women receiving STEM doctorate degrees has been at or above $40 \%$ over the last 15 -plus years [13].

Many universities across the U.S. have taken active steps to improve faculty diversity and the advancement of women and faculty of color. The National Science Foundation (NSF) has played an important role in this regard through its NSF ADVANCE Program. This is a funding program aimed at understanding and addressing gender-based inequities - and their interactions with race/ethnicity - in academic STEM fields. The University of X (UX) has received two NSF ADVANCE grants. The first is a smaller PAID grant to develop workshops and resources on faculty recruitment and on formal mentoring for engineering and the natural sciences at UX.4 The second is an ADVANCE Institutional Transformation (IT) grant. This is a larger award with a broader range of initiatives and objectives than those of the PAID grant. Work done under this grant involves empirical research on institutional structures, practices, and policies that support or inhibit faculty diversity and success at UX. Examples include practices and policies surrounding recruitment, mentoring, promotion \& tenure, workload, and departmental climates.

In this paper, we discuss efforts to better understand faculty retention at UX. We are interested not only in whether there are disparities in retention by gender; we also aim to understand some underlying factors driving faculty departures. Preliminary analyses are quantitative. We examine the rates of departure among different demographic groups over a

1. STEM' refers to the biological, natural, physical, health, engineering and mathematical sciences.

2 These data represent the proportion of women by tenure status of science, engineering and health doctorate holders employed in U.S. universities in 2017.

3 Ibid

4 'PAID' stands for 'Partnership, Adaptation, Implementation, and Dissemination'. 
seven-year time window and find disparities by gender, race/ethnicity, and discipline. We follow up with a cohort analysis of faculty hired or promoted within the same three-year window and, again, find differences by race/ethnicity, gender, and discipline.

The second stage of our analysis is qualitative. Knowing that faculty from different demographic groups depart at higher rates than relevant faculty counterparts may point to potential inequities at the institution. It does not, however, tell us why faculty leave or how to improve faculty retention at UX. For this, we interviewed a sample of faculty who have left UX and a sample who have received outside offers but have been retained over the last five years. Semi-structured interviews covered areas of professional (dis)satisfaction as well as factors that actively contribute to faculty turnover decisions. Although our findings are discussed in detail below, we note that institutional decision making is a primary factor driving turnover for both genders. Yet, the way it matters differs for men and for women. Male faculty are largely concerned with the relationship between access to professional resources and institutional decision making. Women, on the other hand, connect institutional decision making to a broader range of factors including resource distribution, departmental climate, and mentoring. After presenting these findings, we discuss some of the steps that UX is taking to promote improved faculty retention and satisfaction.

It is important to note that the findings that we describe in this paper represent a work in progress. They are part of a larger research project on faculty retention. Follow-up interviews and analysis are underway to help us gain a better understanding of the scope of our findings.

\section{Quantitative Analysis and Findings}

Much of the literature on faculty retention relies on turnover intentions (i.e., plans or desires to leave) as a proxy for actual turnover [2], [5], [8], [12], [18], [20]. Very little of this work examines faculty retention directly. Of those that do, samples are often constructed from college catalogues and online resources [1], [10]. Our study differs from previous research by examining actual faculty departures and retention cases with the use of a comprehensive data set provided by UX's Office of Institutional Research. In contrast to catalog data and online resources, institutional data sets are more reliable and allow for removal of retired faculty.

In the first stage of our quantitative analysis, we examined faculty retention over a sevenyear window by gender, discipline, rank, and race/ethnicity. Our sample $(\mathrm{N}=72)$ consisted of all faculty who departed UX between 2011 and 2017. The demographics of this sample are presented in Tables 1 and 2 .

Results suggest gender differences in retention overall and within the subgroup of STEM faculty. Across all ranks and disciplines, the percentage of women faculty departures (47.2\%) outpaces their overall representation $(36.1 \%)$ at UX. Likewise women STEM T/TT faculty departures (also 47.2\%) outpaces their overall representation (29.3\%) among STEM T/TT. 
Our data also suggest, not surprisingly, that faculty are more likely to depart pre-tenure than post-tenure. In the 7-year period under investigation, UX lost $62.5 \%$ of its TT faculty as compared with $37.5 \%$ of its tenured faculty. No gender differences are observed in the departure rates of TT faculty. However, among tenured associate and full professors, women's departure rates $(44.4 \%)$, once again, exceed their overall representation $(32.7 \%)$ at the university.

While the number of non-white faculty in the sample - and in faculty positions at UX are small, our findings also suggest retention differences by race/ethnicity. In particular, white faculty are being retained at higher rates and black faculty departures are outpacing other racial/ethnic groups. Whites make up $58.3 \%$ of all faculty departures, which is well below their representation at UX (78.6\%). By comparison, black faculty make up $15.3 \%$ of all departures, despite the fact that they represent only $4.3 \%$ of all UX faculty. To put this in perspective, it helps to note that the number of black faculty departures $(\mathrm{N}=11)$ is slightly higher than the number of Asian faculty departures $(\mathrm{N}=9)$. Yet, the average number of Asian T/TT faculty $(\mathrm{N} \approx$ $120)$ is well above the average number of black T/TT faculty $(\mathrm{N} \approx 39)$ over the 7 -year time window under consideration. When examining the intersection between race and gender, we noticed that black female $(\mathrm{N}=8)$ faculty depart in higher proportions relative to their black male counterparts $(\mathrm{N}=3)$. The Asian faculty departures show the opposite pattern (with 3 female and 6 male departures). No other racial/ethnic differences were observed.

\begin{tabular}{|l|c|c|c|}
\hline & Total, M\&F & Total F & $\mathbf{\%}$ \\
\hline All Ranks & 72 & 34 & $47.2 \%$ \\
\hline Tenured & 27 & 12 & $44.4 \%$ \\
\hline Non-Tenured, On-Track & 45 & 22 & $48.9 \%$ \\
\hline \multicolumn{4}{|l|}{ Table 1. Departures, T/TT Faculty, 2011-2017 by Gender } \\
\hline
\end{tabular}

\begin{tabular}{|l|c|c|}
\hline IPEDS Race/Ethnicity & Total \# & \% \\
\hline Hispanic & 3 & $4.2 \%$ \\
\hline Black & 11 & $15.3 \%$ \\
\hline White & 42 & $58.3 \%$ \\
\hline Asian & 9 & $12.5 \%$ \\
\hline Other (Multi/International) & 7 & $9.7 \%$ \\
\hline All R/E Groups & 72 & $100 \%$ \\
\hline
\end{tabular}

Table 2. Departures, T/TT Faculty 2011-2017 by Race/Ethnicity 
One limitation of the above analysis is that it examines faculty retention over a fairly large time-window (7 years). As with any institution, there were changes in environmental conditions - such as changes in leadership, budget model, and institutional culture - during that window. To better control for such changes, we obtained a list of career outcomes for all full time T/TT faculty hired between 2008 and 2010. We used these data to conduct cohort analyses comparing retention and promotion outcomes by rank and gender for the 2017-2018 academic year.

As with the first stage of quantitative analysis, the second stage reveals gender differences in retention. Among the cohort of TT assistant professors $(\mathrm{N}=70)$, a larger percent of women are promoted and retained than men (see Figure 1). This result differs from the findings described above, which suggest no meaningful gender differences in departure pretenure. However, the results for tenured associate professors are consistent with the above findings. Among the cohort of faculty whose first year as associate professor occurred from 2008-2010, women are less likely to be promoted to full professor and are more likely to depart UX. After 8-10 years in rank, $54 \%$ of the men and $30 \%$ of women received promotion to full professor. Likewise, only $1.9 \%$ of the men and $12.2 \%$ of the women had left UX by 2018 . We find similar results within the cohort of tenured associate professors in the STEM disciplines (see Figure 2).

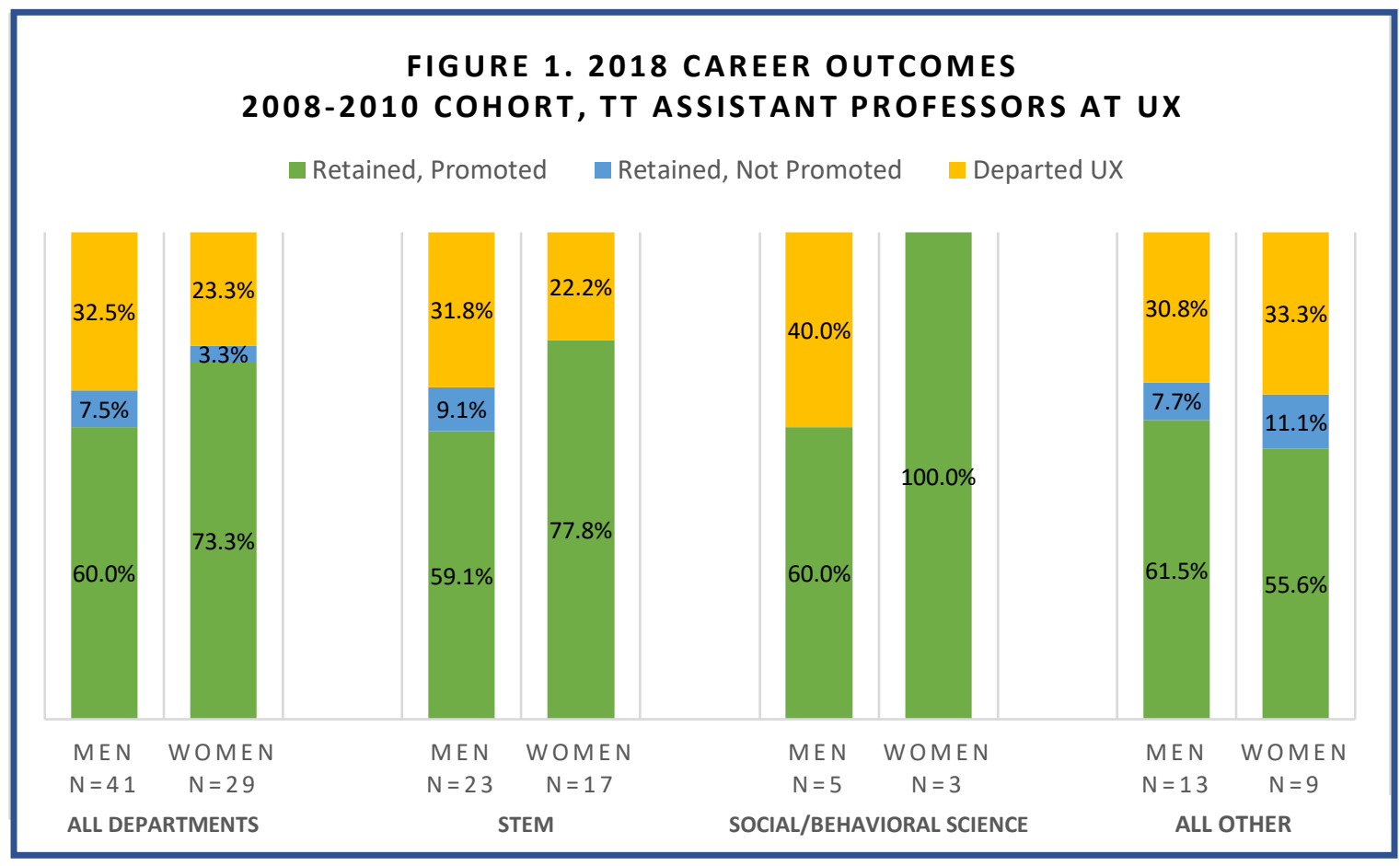




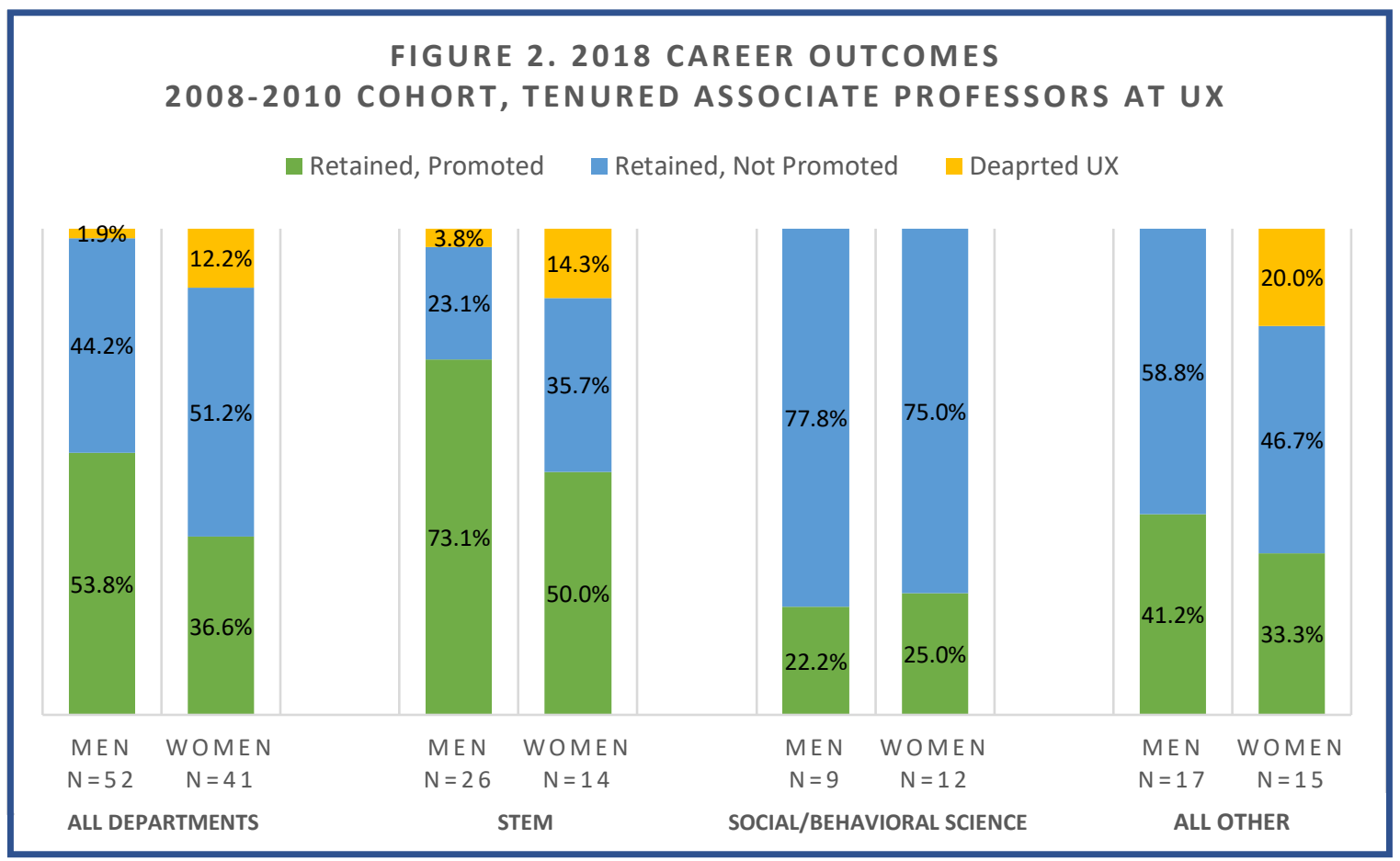

\section{Qualitative Analysis and Findings}

The second stage of our analysis goes beyond an examination of facuıy ıumunun patterns by seeking to understand the underlying factors that drive faculty turnover. Previous studies have linked faculty career satisfaction to turnover intentions, frequently using turnover intentions as a proxy for actual departures [2], [9]. A key finding is that women faculty have lower levels of job satisfaction and higher turnover intentions than their male colleagues [2], [3], [4], [5], [8], [12], [18], [20]. Building from this literature, we chose to examine actual faculty departures instead of turnover intentions, and conducted semi-structured interviews with a sample of faculty who had recently departed UX.

To generate our interview sample, we worked with administrators and the office of institutional research to compile a list of full-time faculty who departed UX from 2015-2019. Faculty retirements were excluded from the sample. From this list, we identified current contact information for a total of 29 faculty. These faculty were asked to participate in the study via email; follow-up emails were sent to those who did not respond to the first invitation to participate. A total of $12 \mathrm{~T} / \mathrm{TT}$ faculty agreed to be interviewed (41\% participation rate), including 6 men and 6 women. All six of the male faculty identified as white, while the female faculty included one white woman and five women of color (black, Latina, and multi-ethnic). Of these exit cases, one was non-voluntary - e.g. a tenure denial. The remaining cases can be categorized as voluntary turnover. 
Most interviews were conducted in 2018 and 2019. The interviews began with questions about respondents' work history at UX, their employment position after leaving the university, and factors they found most appealing about the new position. The next topics covered included areas of general satisfaction and dissatisfaction at UX, such as climate, salary, access to resources, institutional decision making and leadership, promotion \& career development, and work-life. The interviews concluded with questions related to the decision to leave UX, including the factors that played a role in the decision to leave and a detailed discussion of the process (e.g. job search, notifying chair/dean of outside offer, any subsequent retention efforts). Interviews ranged from 20 to 60 minutes, were conducted in person or over the phone, and were audio recorded and transcribed. De-identified interview transcripts were coded in NVivo. The research team first read 3-5 transcripts each and generated a list of codes encompassing the work experiences, both positive and negative, described by our respondents. After a first round of preliminary coding, a second round of coding was undertaken to refine the code list, generating new codes as necessary and collapsing codes that overlapped. The themes described below emerged inductively from this coding process.

It is noteworthy that our exit interview sample over-represents women of color (black, Latina, and muti-ethnic women) and white males. The over-representation of women of color may not have been an accident given the proportional over-representation of women of color departures relative to other demographic groups over the last seven years (as described in the previous section). It follows that when we discuss the perceptions and experiences of women faculty who have departed, it is largely about women of color. And, of course, when discussing the perceptions and experiences of male faculty who have departed, it is largely about white males. The reader should also keep in mind that the experiences of faculty who have departed may differ from those who remain at UX. It is, therefore, an open question whether and to what extent the perceptions and experiences of faculty who have exited UX are reflective of the broader population of faculty at UX.

\section{Why Faculty Leave}

Faculty who leave their institutions do so for a variety of reasons. Some of these factors relate to the appeal of the new institution - such as the promise of a higher salary, academic prestige, or professional opportunities. Other factors relate to areas of dissatisfaction with the current institution. We refer to these as "pull" and "push" factors respectively. This "pull" and "push" framework emerged during data analysis. Unlike a survey, where you might ask a faculty respondent to identify the primary reason they left an institution, we constructed our interview protocol to gather rich data about faculty experiences and explore the process by which faculty came to leave the university. In speaking about their experiences at UX, respondents highlighted what we characterize as push factors, or elements of relative dissatisfaction that played a meaningful role in their decision to leave. They also spoke of pull factors, or aspects of their new institution that were particularly appealing. As we describe later in the paper, for the majority of 
respondents "pull" factors did not play as large a role in the decision to leave as did "push" factors.

By constructing our interview protocol to encourage broader discussion of professional experiences and areas of relative satisfaction and dissatisfaction, faculty provided detailed explanations as to the factors that emerged as key in their decision to leave the institution. In our subsequent analysis, we focus largely on areas of dissatisfaction rather than satisfaction. Respondents offered much more detailed accounts of these areas of dissatisfaction, often comparing their experiences at UX to that of their new institution. This is not surprising, given that the faculty we interviewed had all chosen to leave the university. That being said, it is important to note that in this paper we do not focus on what faculty enjoyed about their professional life at UX. Rather, we focus on the areas they identified as needing improvement, paying particular attention to the factors that emerged as playing a meaningful role in their decision to leave.

In what follows, we first discuss the factors that women faculty who exited cite as areas of dissatisfaction (push factors) and compare these results to those of men who have exited. This is followed by a discussion of factors that faculty cite as appealing about their new position (pull factors) and analyze the interplay between push and pull factors. We conclude that the push factors may be more important than the pull factors in understanding faculty departures at UX. The quotes that we provide are not exhaustive; we rely on comments that best represent the perceptions and experiences of a number of respondents.

\section{Push Factors: Areas of Dissatisfaction for Women}

Women faculty who have left $\mathrm{UX}(\mathrm{N}=6)$ expressed dissatisfaction across a range of areas. The most common area of dissatisfaction related to institutional decision making. Climate and mentoring were also identified but were often linked to institutional decision making. Interestingly, and contrary to what one often reads in the literature, work-life balance did not come up as a key area of dissatisfaction among our female respondents.

\section{Institutional Decision Making}

Nearly all female respondents described how the actions and decisions of department chairs, deans, or other institutional leaders contributed to their dissatisfaction. Concerns ranged from a lack of transparency to perceptions of inequity in the allocation of resources.

Respondent 6, for instance, described what she saw as non-transparent decision making at the departmental level, where decisions were made and communicated to faculty only after the fact. She connected this lack of transparency to the leadership style of her department chair. Her chair "wanted to give the faculty input all the time ...He didn't want [any] decision to be clearly his." While one might assume this consensus-building leadership style would lead to transparency 
and equity in decision-making, it seemed to have an unintended consequence whereby decisions made by the faculty at departmental meetings would suddenly change because someone felt empowered to have a quiet discussion with the chair outside of the departmental meeting.

\section{So...even though there was this illusion that there was a faculty coming together. I think a lot of decisions were made behind closed doors, with small groups of people. (R6)}

Similarly, Respondent 30 expressed that UX suffers from an absence of strategic planning, haphazard decision making, and a lack of transparency. In her view, "way too many" people have been at the university for their entire careers, leading to an insular group of decision makers where "everybody meets at the gym." She coined decision making at the university as "policy by personality" and described the differences between her previous institution and the university: "I actually missed the bureaucracy of [my previous employer], which I never thought I would say. ... At [this university decisions seem random] and there's no transparency..”

Another common element of dissatisfaction with institutional decision making stems from perceived inequities in resource allocation, such as lab space and research budgets. For instance, Respondent 22 explained that prior to assuming an administrative role, she had been largely unaware of the departmental budget, but became "disturbed" by the lack of equity in departmental allocations, citing that there were clearly departments within the college that were more favored than others.

While experiences of inequities in resource allocation varied by respondent, a theme across multiple cases was the lack of follow-through on promises made. As we will see below, lack of promise keeping was also a concern expressed by men who have exited UX. For Respondent 6, her research required certain lab space and equipment. She was happy with the start-up package she had negotiated. However promises of resources, particularly research space, made when she was recruited were continually deferred: "the main reason why I chose to leave $[\mathrm{UX}]$ is because I was promised certain things during my interview that switched completely after I turned in my contract." What was even more demoralizing was that though space constraints were communicated as being severe, the chair was able to accomodate the space needs of another faculty member.

\section{Climate}

A second area of dissatisfaction among the majority of women respondents relates to departmental climate. As with leadership and decision making concerns, particular experiences vary by respondent but the overall pattern is one of non-inclusive departmental climates. For example, Respondent 6 shared that there was a negative climate for women, especially at faculty meetings: 
I noticed that when the other two women would say something, there was no acknowledgment. There was no support. There just seemed to be just a negative attitude in the room...There just seemed to be a very male-dominated atmosphere in that department. (R6)

She felt it was a "strange department" overall as far as climate: "people are friendly and nice, but that doesn't really mean anything- in some cases, it's not really genuine." Similarly, Respondent 29 reported that while her colleagues were pleasant enough in everyday interactions and "generally speaking, it was a collegial group" she did not have close relationships with any of them. She had chosen not to live close to campus, which she perceived as causing negative reactions from colleagues.

I never felt that I was an insider...I just felt like [colleagues] perhaps felt I wasn't as committed. The level of support [I got] perhaps wasn't as strong as others may have gotten who were closer, lived closer. I think maybe there was always some sort of question mark whether she's really going to stay here, that sort of thing. (R29)

Respondent 29 also felt as though her colleagues could have been more professional during the tenure process.

It is noteworthy that several female faculty linked perceptions of negative climate back to dissatisfaction with institutional decision making. Respondent 25 described a departmental colleague who had recently left the university after experiencing conflict with a department chair: "the chair is particularly abusive, and I have seen the university do nothing about it. Nothing." When asked to further elaborate on the climate of the department, Respondent 25 explained:

It didn't have a very tight culture. It didn't have a cohesive culture.........because of a lack of leadership, and it was very intimidating. I would say that .... a lot of faculty members felt that. It wasn't just me. (R25)

Respondent 25 felt as though this climate issue was directly linked to the leadership style of the department chair. She felt the chair did not react positively to faculty advocating for change or disagreeing with decisions. This led to faculty feeling largely "disempowered and intimidated."

In another case, Respondent 22 generally liked her department but felt that departmental, college, and university leadership would frequently "undermine" this climate. She had close relationships with two colleagues who recently left the university and "they both felt very strongly that they had contributed significantly to the university and [yet based on leadership actions felt] that they didn't matter to the university." Respondent 22 was also aware of instances of inappropriate 
conduct and interpersonal conflict between faculty in her department. She had tried to address climate concerns multiple times, but found her concerns did not generate action: "I went to HR many times, made formal complaints... and I sought guidance, actually. I asked for team building resources." In a similar case, Respondent 30 described actions by administrators that she viewed as unprofessional and at times hostile, but were nevertheless allowed to continue by more senior leaders.

\section{Mentoring}

Half of the female respondents described elements of their experiences that indicated a lack of effective mentoring. In the case of Respondent 29, tenure and promotion processes were particularly salient. During her time at UX, there were changes in expectations about the type of scholarship needed for tenure:

The department sort of changed its perspective on tenure and was trying to ramp it up from where it was when I first joined the group. And the tenure requirements were never formally modified in the [departmental] document. But it was sort of an implicit understanding that the school, the department, was trying to make the tenure requirements more strident (R29)

This was especially challenging due to the new expectations not being formalized, and changing during her time as an assistant professor: "you need to have some sort of grandfathering, so that people who came in under a different set of expectations are not penalized." Respondent 29 perceived there were unwritten expectations that did not align with formal tenure documents, but did not have the mentoring or informal networks of colleagues to help navigate these inconsistent expectations. She felt as though access to "unwritten policies and understandings" might have been easier for majority group peers: "that's where women and minorities really lose out. Because you have to be an insider to really know some of those unwritten understandings, right?" Respondent 29 also did not feel as though she received helpful advice from her formal mentor or from her department chair. Based on her experience, she thought the mentoring offered in her department should be improved:

The mentors should be making sure that you are moving along and keeping his or her ear on the ground for you. And so, it's not just, oh, how were your holidays? Oh, did you get that new computer? And that's good. But there should be an explicit focus on advancement and promotion and how to make that happen. (R29)

The sentiment that promotion and tenure standards had changed and that junior faculty were not receiving adequate mentoring to help them be successful was shared by other respondents. 
Respondent 25 was concerned about inequities in promotion and tenure processes, including shifting standards within the department and the lack of mentoring for faculty of color.

Even for more senior female faculty, a lack of professional development and support was notable. Respondent 22 found that her chair was not providing mentoring and professional development during annual reviews. "There was not a discussion about my role in the department or my professional growth, not even strategizing." She was a tenured faculty member with a successful research program and grant funding, but was looking for guidance on furthering her career and advancing into leadership opportunities. In her view, her chair and other faculty seemed happy with the funding she was bringing in, but otherwise "I think they left me alone is really what they did."

\section{Push Factors: Areas of Dissatisfaction for Men}

\section{Institutional Decision Making}

For men who have left UX (N=6), dissatisfaction with institutional decision making was also the most common theme that emerged from our interviews. Several respondents cited a lack of transparency and inclusive decision making as particularly problematic. Respondent 23 explained that the decision-making process is not transparent, which ultimately hurts departments and their faculty: "My sense is that there is a lot of back room politicking that goes on at the administrative level at this university. And, I think that does not work well in terms of how you make the university function." Respondent 23 was able to point to specific actions that were "highly questionable if not unethical" but shielded from scrutiny.

Unlike female faculty, who linked dissatisfaction with institutional decision making to a range of other negative employment conditions such as departmental climate and mentoring, male faculty largely limited their discussion of leadership challenges to contributing to a lack of key resources needed for professional success. Respondent 8 , for instance, was unhappy with his lab space: "I was probably running the largest research group in the department, and there were people with three times the space that I had, and that was really just for historical reasons." He broached the subject of this inequity with leadership in his college, but was unable to reach a resolution. Similarly, Respondent 24 was not pleased with the leadership of his chair, particularly around an issue of appropriate workspace that caused legitimate safety concerns: "he was a very nice person, but he was avoidant of conflict, which can be problematic in asserting different needs of the department." When Respondent 24 approached his chair about fixing the space issue, he listened but asked Respondent 24 not to escalate the problem, and did not offer a solution: "and I suspect it was a financial problem. He just didn't have the financial resources to do a major renovation of the [space] at that time." 
For Respondent 23, a department chair, this pattern of leadership behavior began as soon as he was recruited to join the university when he received promises of resources that never materialized:

I was assured that we would have a certain number of hires that would take place during the time that I was chair and that never came to fruition...we had some retirements and other faculty losses that ended up shrinking the department. And I think that was a challenge for the department in terms of being able to maintain its vitality and its visibility professionally as well as just doing its daily work. (R23)

This pattern of undelivered promises and lacking resources emerged in other cases, including several situations our respondents shared detailing the experiences of colleagues with similar concerns. While respondents did not have a strong sense for what might be driving these cases of unmet needs for resources, several respondents mentioned a lack of clearly defined priorities and strategic planning at the university and college level.

While nearly all of the female respondents cited climate as an area of dissatisfaction, climate was only raised by a single male respondent. In keeping with our findings for women, there was a clear link between leadership concerns and perceptions of negative climate for faculty. However, for Respondent 23, climate concerns were not strongly linked to his decision to leave the university. In fact, he felt the climate within his department was quite good, with "great colleagues...and a very collegial department." Rather, he had a more general observation that the climate at the university used to be "very good," and the university had been " one of the better places to work among organizations in higher education," but had declined over time. He noted this decline could be attributed, at least in part, to a new leader who seemed to view the university more as a business than an institution of higher learning, explaining that "I think that created a climate that was not well-received by the faculty."

For Respondent 23, declining climate for faculty was directly linked to university administrators, whose priorities and lack of transparency in decision making led to a lack of "responsibility and integrity in terms of how the university is run." Thus university leadership, and lack of follow-through on promised resources, was a stronger driver in his decision to leave the university.

\section{Factors Guiding the Decision to Leave the University}

Over the course of our interviews, respondents spoke at length about the exit process, beginning with when they first began to consider leaving the university through retention efforts and their final decision to leave. A common thread across these narratives was that "pull" factors did not play as large a role in the decision to leave as "push" factors. For women, pull factors, or factors that were most appealing when considering their new institution included (1) availability of 
resources, (2) perceptions of better leadership at the new institution, and (3) dual career considerations. The most common of these factors was the availability of resources. For those respondents in leadership roles such as department chair, the availability of a larger budget coupled with greater autonomy in how to allocate resources were particularly appealing. For faculty that did not hold leadership roles, the draw of larger and better equipped lab space or more generous research funding was appealing. For men, pull factors at their new institution included (1) availability of resources, (2) opportunities for leadership such as building a new department or institute, (3) perceptions of better leadership at the new institution, and (4) strength of department or better fit with one's research program. The most common of these were access to resources (and colleagues) to support research and opportunities for leadership, broadly defined. It is interesting to note that while "push" factors varied by gender, we saw relatively small differences by gender in "pull" factors at other institutions.

As our respondents described the process by which they decided to leave UX, we discovered that "push" factors played a main role in the decision to leave. A small number of respondents, including both men and women, described leaving the university for a particular, targeted opportunity, such as a position at a school in a location close to family or with career opportunities for a partner. These faculty were generally more satisfied with their current position at the university, with the exception of the "pull" factor, than were other faculty who exited. For the majority of respondents, however, it was their current dissatisfaction with the university and its leadership that triggered the choice to leave. Several of these respondents described evaluating a range of institutions before exiting, only choosing to leave once they had identified a particularly good fit at another university. Another group of respondents initially appeared uninterested in leaving, even after being recruited by multiple schools. However, over time, and perhaps as their areas of dissatisfaction with the university were not addressed, they increasingly began to consider options for leaving.

An example of this gradual decision to leave is Respondent 8. He was unhappy with his lab space as compared to peers and raised this concern with leadership in his college to no avail. More broadly, Respondent 8 was displeased with his relationship with a college administrator, who he perceived as having a "business management" style and focus on asking departments to take on additional responsibilities without "resources to support the shift." Over a period of months, as Respondent 8 was frustrated about his own access to resources as well as the allocation of resources in his college, he was recruited by several institutions. After considering these positions, he ultimately felt they were not the right fit and declined to leave. Shortly thereafter he was approached by a senior administrator at another institution. Given his dissatisfaction at the university, Respondent 8 felt "I'm kicking tires, I might as well kick one more tire." After careful consideration, he found himself impressed with the new institution and ready to leave. In his new role he has a lab that is twice the size of the one he left, as well as a better relationship with college and university leadership. This case points to the importance of dissatisfaction in driving exits, as Respondent 8 was not actively looking to leave the institution, 
but became more willing to entertain outside offers as his frustration with UX leadership increased.

Another example of the balance of push and pull factors comes from Respondent 25. This is a case where the faculty member had a high research profile and had been recruited by multiple schools. She initially was not persuaded to leave, but in the end decided to leave UX due in large part to poor working conditions (including inadequate space) and feeling underappreciated by a chair whose leadership style had catalyzed the departure of a colleague. In her view, if her professional satisfaction at UX had been higher, she would have been "fully vested" and more hesitant to leave. As Respondent 25 recounted the process of considering her external job offers, she elaborated that her decision was not simply about resources, salary, or other "pull" factors. If her working conditions at UX had been more positive she would be "willing to take a lower salary- because it's not just about the money. It's about the experience and how vested you are, and I think that I was not." Given the "push" factors she encountered at UX, a generous outside offer including a higher salary and research budget was all the more appealing. Even given her areas of dissatisfaction with UX, Respondent 25 shared that she would have been open to negotiating a retention package with her dean, but felt as though her chair had hindered this effort.

It should be noted that the majority of respondents we interviewed were offered retention packages. These retention offers varied, with some matching or exceeding the salary of the outside offer, while others were not competitive with what the new institution offered. As part of our larger research project we have conducted interviews with faculty who have been retained at UX. While this research is ongoing, preliminary analysis comparing the 12 faculty who exited to 14 faculty who were retained, indicated retention offers were largely similar. However, it should be noted that the faculty who were retained were generally more satisfied with the university to begin with, making them more likely to accept a retention package than faculty with longstanding areas of dissatisfaction. This difference is illustrated well in the case of Respondent 6 , who was frustrated by not getting the lab space and equipment she had been promised when recruited to the university. While her retention offer included these resources, she was understandably skeptical about trusting that these would be delivered: "I couldn't trust [the retention offer]. Especially since this is almost the same language - a little bit less committed than my first interview...It's like, I'm not going to fall for this twice."

\section{Discussion \& Conclusion}

We have examined faculty retention at a single research intensive university using a number of data sources and methods of analysis. As noted earlier, much of the literature on faculty retention in higher education utilizes turnover intentions as a proxy for actual exit behavior [2], [5], [8], [12], [18], [20]. Our study, however, examines actual faculty departures both quantitatively and qualitatively. Of those studies that do examine actual departures, very few report on race/ethnicity. 
Our quantitative findings are interesting for a number of reasons. Recent studies on actual retention patterns have found relative gender parity in faculty turnover [1], [10]. Our results, however, present a somewhat different story. Although men and women have departed UX in roughly equal proportions, the proportion of female departures is higher than their overall representation at UX. Importantly, it is largely the post-tenure women who are contributing to observed gender differences in retention/attrition. It is possible that observed disparities are less about differential potential for professional success and more about other types of factors. Our findings regarding race/ethnicity ought to be taken with caution, since $\mathrm{N}$ here is relatively small, but do indicate reason to be concerned about black faculty and women of color in particular.

Our qualitative analysis of the experiences of faculty who exited the university suggests that effectiveness of institutional decision making and leadership (chairs, deans, and other leaders) is the most important factor driving dissatisfaction and faculty departures. Men and women express dissatisfaction with institutional decision making and leadership at similar rates, but experience the importance of leadership in different ways. For men, dissatisfaction with leadership is also linked to unhappiness with resources such as lab space, equipment, or funding. For women, dissatisfaction with leadership is linked to unhappiness with resources, climate, and mentoring. This gender difference aligns with research that finds female faculty perceive less institutional support and recognition than male faculty. It suggests that improved relationships with department chairs may be one avenue by which women faculty can access institutional support [15], [22]. Interestingly, we find that work-life balance may not be a common driver of female faculty departures. This result runs counter to the narrative that identifies family related concerns as an important factor in explaining the leaky pipeline for women in academic careers [6], [11], [12], [21].

Our results also point to the primary importance of "push" factors relative to "pull" factors in driving faculty turnover. Faculty are not likely to leave an institution simply for a higher salary or increased prestige (pull factors), unless they are already dissatisfied with their current institution (push factors) [14], [16], [17]. It follows that faculty retention is something that can be enhanced through the active efforts of institutional leaders.

While this paper focuses on the experiences of faculty who have exited UX, the broader research project that is now underway includes interviews with retained faculty (e.g. those that received outside offers and negotiated retention packages) and a matched sample of current faculty. Findings comparing the experiences of these different populations will be discussed in future publications.

\section{Impact on ADVANCE Work}

The ADVANCE team at UX will use these findings to inform activities and initiatives surrounding retention. Guided by research on the importance of leaders as shapers of climate, 
several of our activities are aimed at supporting chairs, deans, and other institutional leaders. We are on the provost calendar every other week and meet regularly with the deans. Meetings are supported by "talking points", which are empirically driven easily digestible facts, figures and best practices for faculty diversity and success. We also have a number of chair mentoring activities, workshops and networking events. As we continue these activities, particular attention will be paid to departmental climates, equitable distribution of resources, faculty mentoring, and transparency of policies and procedures.

Our findings also underscore the importance of ADVANCE efforts surrounding faculty mentoring. In partnership with the UX Faculty Senate and the Provost's office, UX ADVANCE played a role in requiring departments to develop formal mentoring policies. To support these efforts, we developed workshops and resources on formal mentoring for mentors and mentees. To date, however, most mentoring efforts have targeted assistant professors. Given that gender differences in retention occur largely at the post-tenure level, there is a need for increased efforts to support associate and full professors.

UX ADVANCE also offers a number of programs and events designed to facilitate networking and professional development for women faculty. These programs encourage faculty to connect with peers, share experiences, and develop strategies for professional success, which may help to address our finding that women feel dissatisfied with access to resources, climate, and mentoring. One example of this type of activity is our leadership program for mid-career female faculty, which launched in 2018. This intensive, small-group program helps women hone leadership skills, develop a personal leadership plan, navigate current work challenges, and build a cohort of women faculty for peer networking and support.

\section{Limitations and Future Work}

As described previously, women of color are overrepresented in our sample of faculty who exited. Given that women of color make up five of the six female exit interviews, while all of male exit interviews are from white males, observed gender differences may be best described as differences in the experiences of women of color as compared to white men. Research is underway to examine the experiences of faculty who were retained, as well as a matched sample of current faculty. This will allow us to better understand the factors that increase the likelihood of faculty being retained (some faculty would like to leave, but are unable to; other faculty may have their concerns addressed during retention offers).

\section{References}

1. J. M. Box-Steffensmeier, et al., "Survival Analysis of Faculty Retention and Promotion in the Social Sciences by Gender," PLOS One, 10, e0143093, 2015. 
2. R. R. Callister, "The Impact of Gender and Department Climate on Job Satisfaction and Intentions to Quit for Faculty in Science and Engineering Fields," Journal of Technology Transfer, vol. 31, pp. 367-375, 2006.

3. C. Daly and J. Dee, "Greener Pastures: Faculty Turnover Intent in Urban Public Universities," Journal of Higher Education, vol. 77, no. 5, pp. 776-803, 2006.

4. F. M. Deutsch and B. Yao, "Gender Differences in Faculty Attrition in the USA," Community, Work \& Family, vol. 17, no. 4, pp. 392-408, 2014.

5. S. K. Gardner, "I Couldn't Wait to Leave the Toxic Environment: A Mixed Methods Study of Women Faculty Satisfaction and Departure from One Research Institution," NASPA Journal About Women in Higher Education, vol. 5, no. 1, pp. 71-95, 2012.

6. M. Goulden, M.A. Mason, and K. Frasch, "Keeping Women in the Science Pipeline," The Annals of the American Academy of Political and Social Science, vol. 638, no. 1, pp. 141$162,2011$.

7. C. Hill, et al., "Barriers and Bias: The Status of Women in Leadership," Special Report. American Association of University of Women, April 2016. Available here: https://files.eric.ed.gov/fulltext/ED585546.pdf

8. S. Hurtado and L. DeAngelo, "Keeping Senior Women at Your College," Academe, vol. 94, no. 5, pp. 18-20.

9. L. K. Johnsrud and V. J. Rosser, "Faculty Member's Morale and their Intention to Leave: A Multilevel Explanation," The Journal of Higher Education, vol. 73, no. 4, pp. 518-542, 2002.

10. D. Kaminski and C. Geisler, "Survival Analysis of Faculty Retention in Science and Engineering," Science, vol. 335, pp. 864-866, February 2012.

11. M. A. Mason, N. H. Wolfinger, and M. Goulden, Do Babies Matter, New Brunswick, NJ: Rutgers University Press, 2013.

12. S. K. McCoy, E. E. Newell, S. K. Gardner, "Seeking Balance: The Importance of Environmental Conditions in Men and Women Faculty's Well-Being," Innovative Higher Education, vol. 38, pp. 309-322 2013.

13. National Science Foundation, National Center for Science and Engineering Statistics, "Women, Minorities and Persons with Disabilities in Science and Engineering: 2019," Special Report. NSF 19-304, Alexandria VA, 2019.

14. M. W. Matier, "Retaining Faculty: A Tale of Two Campuses," Research in Higher Education, vol. 31, pp. 39-61, 1990.

15. D. Olsen, S. A. Maple, and F. K. Stage, "Women and Minority Faculty Job Satisfaction: Professional Role Interests, Professional Satisfactions, and Institutional Fit," The Journal of Higher Education, vol. 63, no. 3, pp. 267-293.

16. K. A. O’Meara, “Half-Way Out: How Requiring Outside Offers to Raise Salaries Influences Faculty Retention and Organizational Commitment," Research in Higher Education, vol. 55, no. 4, pp. 1-22, 2014. 
17. K. A. O’Meara, et al., "Left Unsaid: The Role of Work Expectations and Psychological Contracts in Faculty Careers and Departure," The Review of Higher Education, vol. 39, no. 2, pp. 269-297, 2016.

18. V. J. Rosser, "Faculty Member's Intentions to Leave: A National Study on Their Worklife Satisfaction," Research in Higher Education, vol. 45, pp. 285-309, 2004.

19. S. Shepherd, "Why Are There So Few Female Leaders in Higher Education: A Case of Structure or Agency?," Management in Education, vol. 31, no. 2, pp. 82-87, 2017.

20. C. Turner, J. González, and J. Luke Wood, "Faculty of Color in Academe: What 20 Years of Literature Tells Us," Journal of Diversity in Higher Education, vol. 1, no. 3, pp. 139-168, 2008.

21. Ward, Kelly, and Lisa E. Wolf-Wendel. "Work and Family Perspectives from Research University Faculty." New Directions for Higher Education, vol. 130, pp. 67-80, 2005.

22. Y. J. Xu, "Gender Disparity in STEM Disciplines: A Study of Faculty Attrition and Turnover Intentions," Research in Higher Education, vol. 49, no. 7, pp. 607-624, 2008.

23. B. L. Yoder, "Engineering by the Numbers," American Society for Engineering Education, 2019. 